\title{
IRF4 selectively controls cytokine gene expression in chronic intestinal inflammation
}

\section{Jonas Mudter ${ }^{1}, 2$, 3 , Jingling $\mathbf{Y u}^{2}$, Lioubov Amoussina², Benno Weigmann², Arthur Hoffman ${ }^{3}$, Katrin Rücknagel ${ }^{3}$, Peter R. Galle ${ }^{3}$ and Markus F. Neurath', 2, 3}

\author{
${ }^{1}$ 1st Medical Clinic, University of Erlangen-Nürnberg, Germany \\ ${ }^{2}$ Institute for Molecular Medicine, University of Mainz, Germany \\ ${ }^{3} 1$ st Medical Clinic, University of Mainz, Germany
}

Received: 2008.12.17, Accepted: 2009.03.06, Published online: 2009.08.20

(c) L. Hirszfeld Institute of Immunology and Experimental Therapy, Wrocław, Poland 2009

\begin{abstract}
The authors previously showed that interferon regulatory factor (IRF)4 knockout mice are protected from experimental oxazolone and TNBS colitis. Here the effect of IRF4 on the expression of pro- and anti-inflammatory cytokines in TNBS colitis and long-term $\mathrm{CD}_{45 \mathrm{RB}^{\text {high }}}$ transfer colitis is examined. In TNBS colitis, no differences were found in interleukin (IL)-18 and tumor necrosis factor (TNF)- $\alpha$ expression between IRF4 knockout and wild-type mice. However, significant differences were detected in IL-6 and IL-17 production. Upon treatment with hyper-IL-6, IRF4 $4^{-/}$mice lost their protective properties towards TNBS application. Hyper-IL-6 application induced IL-6 mRNA, but not IL-17 mRNA expression, suggesting that IL-6 deficiency is not primarily responsible for the lack of IL-17 production. T-bet and GATA-3 mRNA expressions were not affected upon IL-6 application. In transfer colitis, colonic cytokine mRNA analysis revealed a reduced production of IL-6 in IRF4 ${ }^{-/}$ reconstituted mice in the long-term course. In contrast, several other cytokines did not differ between the two groups (e.g. TNF- $\alpha$ and IL-10). Measurement of supernatants from splenic mononuclear cells revealed a significant difference in IL-6 and IL-17 production between the two groups. These findings suggest that IRF4 selectively regulates cytokine gene expression in chronic inflammation. IRF4 therefore emerges as an attractive target for the therapy of chronic intestinal inflammation. Blocking IRF4 might be an interesting option to modulate inflammation in the advanced state of inflammation.
\end{abstract}

Key words: IRF4, T cells, interferon regulatory factor, IL-6, IL-17.

Corresponding author: Prof. Markus F. Neurath, Medizinische Klinik 1, Ulmenweg 18, Universität Erlangen-Nürnberg, D-91054 Erlangen, Germany, tel.: +49 9131-853500

\section{INTRODUCTION}

Inflammatory bowel diseases are driven by a mucosal cytokine imbalance resulting from a misled immune response influenced by luminal antigens and a defective mucosal barrier in genetically susceptible individuals (Macdonald and Monteleone 2005; Mudter and Neurath 2003; Podolsky 2002; Sartor 2004; Shanahan 2002; Wehkamp et al. 2005). To characterize the diverse aspects and cytokine interactions of the immune response in intestinal inflammation, several experimental models have been established (Blumberg et al. 1999; Strober et al. 2002). Such in vivo studies have shown that interleukin (IL)-6 signaling plays a pivotal role in inflammatory bowel diseases. In intestinal inflammation, IL-6 transsignaling contributes to enhanced T-cell survival and accumulation of activated $\mathrm{T}$ cells in the lamina propria (Atreya et al. 2000; Mudter et al. 2002).

The transcription factor interferon regulatory factor (IRF) 4 is a member of the IRF family of transcription regulators. Previous in vivo studies of IRF4 function pointed out its critical role in T helper (Th) 2 differentiation (Lohoff et al. 2002). IRF4-deficient (-/-) and IRF4 ${ }^{+/-}$mice were infected with Leishmania major promastigotes in their footpads. One week later, regional lymph node cells were isolated and re-stimulated in vitro. The IRF4 ${ }^{+/}$mice exhibited IL-4 and interferon (IFN) $-\gamma$ secretion. In contrast, lymph node cells from the IRF4 ${ }^{-/}$mice produced neither IL-4 nor IFN- $\gamma$. Furthermore, in vitro differentiation studies showed strongly impaired Th1 and Th2 cytokine responses of $\mathrm{IRF}^{-1-} \mathrm{T}$ cells. It was found that IL-4-dependent Th2 
differentiation was due to the inability of IRF4 $4^{-/-}$lymphocytes to upregulate GATA-3 expression. The ability to undergo Th2 differentiation could be restored by retroviral overexpression of GATA-3. Furthermore, a strong synergism between NFATc2 and IRF4 in IL-4 gene transcription was reported (Rengarajan et al. 2002). Very recently, IRF4 was also attributed to play a role in IL-17 production (Brustle et al. 2007). The authors showed that IRF4-deficient mice did not develop experimental autoimmune encephalomyelitis and that $\mathrm{T}$ helper cells from such mice failed to differentiate into Th17 cells.

In previous studies we could show that IRF4 deficiency protected from experimental colitis due to reduced expression of IL-6. In this study the aim was to analyze the influence of IRF4 deficiency on cytokine expression in long-term colitis.

\section{MATERIALS AND METHODS}

\section{Animals and induction of colitis}

The TNBS colitis model was previously described (Boirivant et al.1998; Neurath et al.1995). Transfer colitis was performed as described by Morrissey et al. Powrie et al. (Morrissey et al. 1993; Powrie et al. 1993). In brief, $\mathrm{CD}^{+}{ }^{+} \mathrm{CD} 45 \mathrm{RB}^{\text {high }} \mathrm{T}$ cells were sorted by flow cytometry (FACScalibur, BD) and $5 \times 10^{5}$ cells were intraperitoneally transferred into $\mathrm{Rag}^{-{ }^{--}}$mice. $\mathrm{T}$ cells were derived either from IRF4 $4^{--}$or C57BL/6 donors. To modulate colitis activity, mice were given $1 \mu \mathrm{g}$ hyper-IL-6 (hyper-IL-6 consists of human IL-6 and the human sIL$-6 \mathrm{R}$ covalently connected by a flexible peptide chain) by intraperitoneal injection (obtained from Prof. Rose-John, University of Kiel, Germany). The injection was made 7 days after skin sensitization with TNBS just before intrarectal administration of the hapten reagents.

\section{Preparation of gut tissues}

The murine colon was divided in a proximal and a distal piece. Colon pieces were snap frozen and either homogenized or embedded in OCT compound to assess cryosections. Paraffin-embedded sections were used to perform hematoxylin and eosin staining for histological scoring. Furthermore, one piece of each part (proximal and distal) was snap-frozen to isolate mRNA.

\section{Histological analysis of colon cross-sections and endoscopy}

Tissues were removed from colitic mice and sections were made and stained with hematoxylin and eosin. The degree of inflammation on the microscopic cross-sections of colon was graded semiquantitatively on a scale of 0-6, or 0-15 in the case of transfer colitis, by the same pathologists blinded to the sample's identity, as described before (Mudter et al. 2008).
To perform experimental endoscopy, the mice were anesthetized using an intraperitoneal injection of avertine (Sigma Chemical Co., St. Louis, MO, USA). Mouse endoscopy was performed using a miniature endoscope (scope outer diameter: $1.9 \mathrm{~mm}$ ), a xenon light source, a triple chip camera, and an air pump (all from Karl Storz, Tuttlingen, Germany). The endoscopic procedure was viewed on a color monitor and digitally recorded on tape (DSR-20MDP; Sony, Cologne, Germany).

Endoscopic colitis scores were based on the observed signs of inflammation. The modified murine endoscopic index of colitis severity consisted of five parameters: thickening of the colon wall, changes in the normal vascular pattern, presence of fibrin, mucosal granularity, and stool consistency. Endoscopic screening was performed for each parameter (score between 0 and 3 ), leading to a cumulative score between 0 and 15 . Healthy mice usually have a score between 0 and 3 (Wirtz et al. 2002).

\section{Cell isolation and purification of spleen mononuclear cells}

Spleen mononuclear cells were isolated from freshly obtained specimens from healthy B6 mice. Erythrocytes were removed from the spleen cell suspension by hypotonic lysis in ammonium chloride and potassium chloride buffer. The MACS system (Miltenyi, Germany) was used to isolate $\mathrm{CD}^{+} \mathrm{T}$ cells. The cells were cultured in complete Roswell Park Memorial Institute medium in a humidified atmosphere with $5 \% \mathrm{CO}_{2}$ in a $37^{\circ} \mathrm{C}$ incubator in the presence or absence of hamster antibodies against mouse CD3 and CD28 (CD3, clone 145-2C11, 2 $\mathrm{mg} / \mathrm{ml}$; CD28, clone 37.51, $0.5 \mathrm{mg} / \mathrm{ml}$; PharMingen, San Diego, CA, USA).

\section{Th17 differentiation}

$1 \times 10^{6} /$ well MACS-purified CD4 $4^{+} \mathrm{T}$ cells were cultured in 24-well plates containing plate-bound anti-CD3 (5 $\mathrm{mg} / \mathrm{ml})$ and soluble anti-CD28 $(1 \mathrm{mg} / \mathrm{ml})$. The cultures were supplemented with $20 \mathrm{ng} / \mathrm{ml} \mathrm{IL-6}$ (eBioscience) or hyper-IL-6, $1 \mathrm{ng} / \mathrm{ml} \mathrm{IL}-1 \beta, 1 \mathrm{ng} / \mathrm{ml}$ tumor necrosis factor (TNF)- $\alpha$, and $5 \mathrm{ng} / \mathrm{ml}$ transforming growth factor- $\beta$ (Preprotech). For flow cytometry and sandwich ELISA the cells were analyzed on day 4.

\section{Cytokine measurement}

The cytokine concentration was measured in the supernatants of either mononuclear spleen cells or isolated $\mathrm{CD} 4^{+} \mathrm{T}$ cells. Quantification was performed using a multiple cytokine measurement kit and flow cytometry according to the manufacturer's instructions (BenderMed Systems). IL-6 and IL-17A were also measured using enzyme-linked immunosorbent assay kits (ELISA Kits; eBioscience, San Diego, CA, USA). The cells were cultured and stimulated for 24 or $48 \mathrm{~h}$ as previously mentioned using anti-CD3 and anti-CD28 (CD3, 
clone 145-2C11, $2 \mu \mathrm{g} / \mathrm{ml}$; CD28, clone 37.51, $0.5 \mu \mathrm{g} / \mathrm{ml}$; PharMingen, San Diego, CA, USA).

\section{RT-PCR and real-time PCR}

A sample of the distal part of the colon was used for RNA isolation. Colonic tissue was disrupted and homogenized by bead-milling in RLT Plus Buffer ${ }^{\circledR}$ (Qiagen, Hamburg, Germany), containing denaturing guanidine-isothiocyanate and $\beta$-mercaptoethanol. RNA was purified by extraction with RNeasy ${ }^{\circledR}$ Plus Mini Kits (Qiagen, Hamburg, Germany).

Reverse transcription (RT) was performed by mixing $1 \mu \mathrm{g}$ of RNA solution, deoxynucleoside triphosphate (dNTP) Mix (PEQLAB Biotechnology GmbH, Erlangen, Germany), random hexamer primer (Invitrogen, Karlsruhe, Germany), and oligo(dT) (MWG-BIOTECH AG, Ebersberg, Germany) and incubating the solution $(13 \mu \mathrm{l})$ for $5 \mathrm{~min}$ at $65^{\circ} \mathrm{C}$. Then 7 $\mu \mathrm{l}$ of a solution containing $5 \mathrm{X}$ First-Strand Buffer (PEQLAB Biotechnology GmbH, Erlangen, Germany), 40 $\mathrm{U}$ of RNaseOUTTM (Eppendorf, Wesseling-Berzdorf, Germany), and dithiothreitol (DTT) (Invitrogen, Karlsruhe, Germany) was added. The mixed contents were incubated for $2 \mathrm{~min}$ at $25^{\circ} \mathrm{C}$. Two hundred units of SuperScript ${ }^{\mathrm{TM}} \mathrm{RT}$ II RT (PEQLAB Biotechnology GmbH, Erlangen, Germany) was added and the tubes were incubated for 10 $\min$ at $25^{\circ} \mathrm{C}$ and then for $50 \mathrm{~min}$ at $42^{\circ} \mathrm{C}$. Finally the tubes were heated to $70^{\circ} \mathrm{C}$ for $15 \mathrm{~min}$. cDNA was mixed with REDTaq $^{\mathrm{TM}}$ ReadyMix ${ }^{\mathrm{TM}}$ PCR Reaction Mix (Sigma-Aldrich CHEMIE Gmbh, Steinheim, Germany) and 24-35 cycles of PCR were performed in a thermal cycler. The PCR products were visualized by agarose gel electrophoresis. Primer pairs were used as previously described (Autenrieth et al. 1997) except that for IL-6 (sense mIL6 e2s GAG AGG AGA CTT CAC AGA GGA T, antisense mIL6 e4a GCT TAG GCA TAA CGC ACT AGG TT).

For real-time PCR, total RNA from a murine colon specimen was extracted using an RNeasy Mini Kit (Qiagen, Hamburg, Germany). One $\mu \mathrm{g}$ of total RNA was reverse transcribed using an oligo-dT primer. Real-time PCR analysis for quantification of the expressions of IL-6, IL-17, GATA-3, and T-bet and $\beta$-actin mRNAs was performed in duplicate on an iCycler (Bio-Rad Laboratories) using the ABsolute SYBR Green Fluorescein Kit (Abgene, Epsom, UK). The primer sets for these experiments were purchased from QuantiTect (Qiagen, Hamburg, Germany). The sequences for IL-17 were: IL-17 sense: GGACTTCCTCCAGAATGT and IL-17 antisense: TCAGGACCAGGATCTCTT. The relative increase in reporter fluorescent dye emission was monitored. The level of cytokine mRNA relative to actin was calculated using the formula:

$$
\begin{gathered}
\text { Relative cytokine mRNA expression }= \\
2 \wedge\left[c_{\mathrm{t}}\left(\text { cytokine }_{\text {control }}\right)-c_{\mathrm{t}}\left(\text { cytokine }_{\text {treated }}\right)+\mathrm{c}_{\mathrm{t}}\left(\text { Actin }_{\text {treated }}\right)-\right. \\
\left.-\mathrm{c}_{\mathrm{t}}\left(\text { Actin }_{\text {control }}\right)\right]
\end{gathered}
$$

where $c_{t}$ is the number of the cycle in which emission exceeds an arbitrarily defined threshold.

\section{Immunohistochemistry}

Immunofluorescence of the cryosections was performed using the TSA Cy3 system (PerkinElmer, Waltham, MA, USA) and a fluorescence microscope (Olympus, Hamburg, Germany) using primary antibodies specific to CD4 (Santa Cruz Biotechnology, Heidelberg, Germany), CD11c, and myeloperoxidase (MPO, BD Biosciences). Before examination, the nuclei were counterstained with Hoechst 3342 (Invitrogen Molecular Probes, Karlsruhe, Germany).

\section{Statistical evaluation}

All experiments were carried out at least in triplicate. The statistical significance of the evaluated data was tested using the Mann-Whitney U-test and Student's $t$-test. Results were considered statistically significant when $\mathrm{p}$ values were $<0.05$. Results are expressed as mean values ( \pm SD or \pm SEM). The error bars in the histograms represent the standard deviations or standard errors of the mean, as indicated.

\section{RESULTS}

Increased IL-6 production in the colons of TNBS-treated

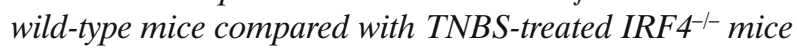

In the first series of experiments we evaluated TNBS colitis. In contrast to the our previously performed TNBS colitis experiments (Mudter et al. 2008), the mice were evaluated on day 5 instead of days 3 and 4 . Thus we sacrificed the mice on day 5. The colon from each mouse was removed and mRNA was isolated to analyze cytokine expression in the IRF4-deficient and wild-type mice using semiquantitative PCR. Interestingly, whereas equal levels of IL-18 mRNA and TNF- $\alpha$ mRNA were detected in the colons of the wild-type and knockout mice, IL-6 mRNA levels in the lamina propria were much higher in the colitic wild-type mice than in the TNBS-treated $\mathrm{IRF}^{-/-}$mice (Fig. 1A).

\section{Administration of hyper-IL-6 exerts a long-lasting proinflammatory effect on colitis activity in IRF4-deficient mice}

To assess whether the reduced mucosal IL-6 production in IRF4 $4^{-/}$mice was relevant in vivo, we next injected the designer protein hyper-IL-6 (IL-6/sIL-6R) intraperitoneally into TNBS-treated IRF4 $4^{-/}$mice (Fig. 1B). This completely blocked the protective effect of IRF4 ${ }^{-/-}$deficiency in TNBS-induced colitis and induced weight loss comparable to that of the TNBS-treated wild-type mice. The effect of hyper-IL-6 was long lasting because the hyper-IL-6-treated colitic IRF4-deficient mice continuously lost weight. In contrast, the TNBS-treated IRF4-deficient mice without hyper-IL-6 treatment gained weight on days 4 and 5. We also performed 
A

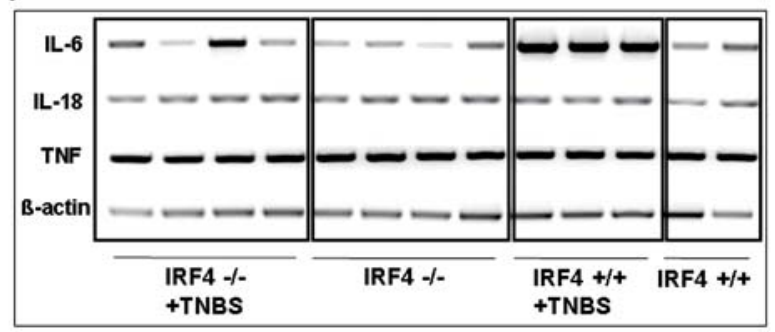

B
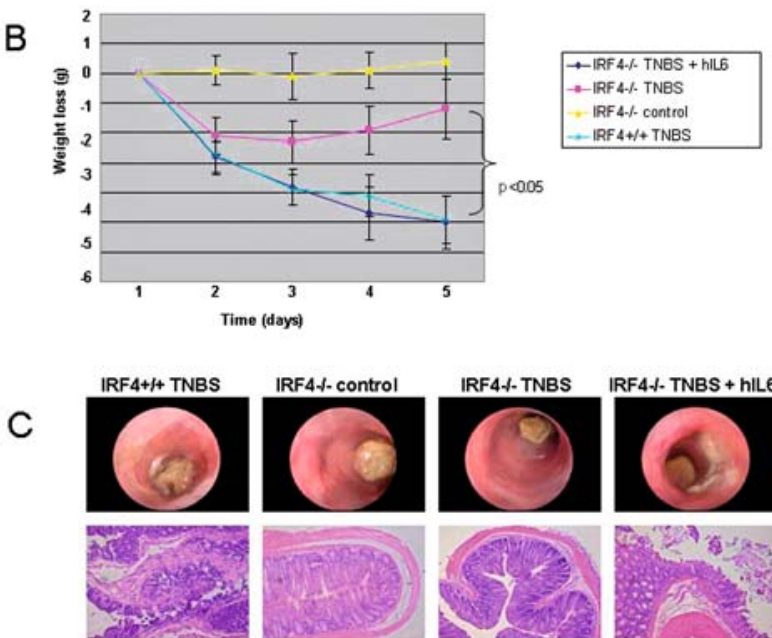
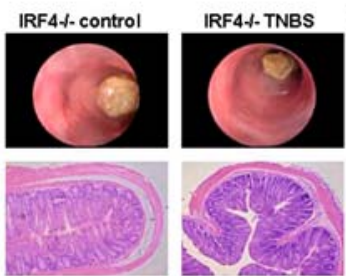

D

Endoscopic score

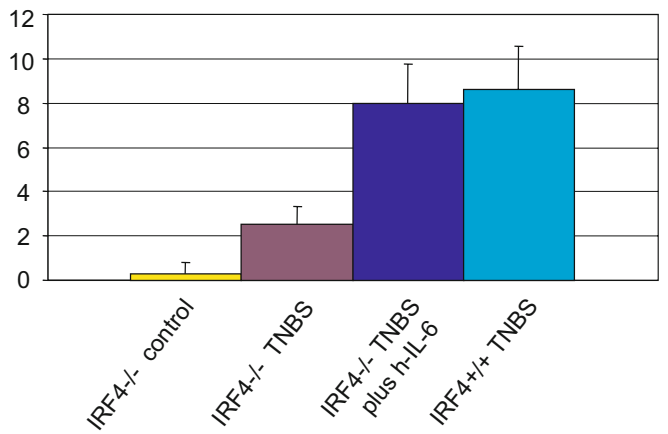

Histologic score

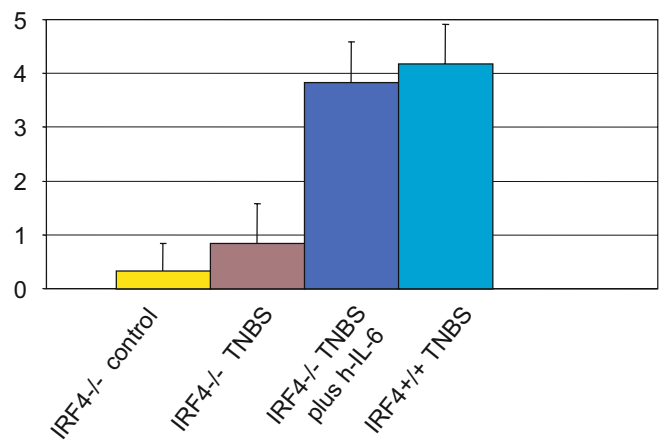

Fig. 1. Evaluation of TNBS coliis. (A) The mRNA expressions of various proinflammatory cytokines in the colons of TNBS-treated and untreated IRF4-deficient and wild-type mice were analyzed by semiquantitative PCR. Whereas no differences in the expressions of the proinflammatory cytokines TNF- $\alpha$ and IL-18 were noted between the groups, IL- 6 mRNA expression was strongly induced in the wild-type, but not in the IRF4-deficient mice upon TNBS administration. (B) IRF4-deficient mice and wild-type control mice were treated by intrarectal administration of the hapten reagent TNBS followed by monitoring of body weight. TNBS-treated IRF4 $4^{-/-}$mice lost significantly $(\mathrm{p}<0.05)$ less weight than the wild-type mice. To determine the functional role of IL-6 signaling for the effects of IRF4 on TNBS colitis activity in vivo, the designer fusion protein hyper-IL-6 (IL-6/sIL-6R) was intraperitoneally administered to TNBS-treated IRF4-deficient mice. This group showed similar weight loss to the TNBS-treated IRF4 $4^{+/+}$mice. One representative experiment out of three is shown $\left(\mathrm{IRF} 4^{+/+}\right.$plus TNBS, $\mathrm{n}=8$; IRF4 ${ }^{-/}$plus TNBS, $\mathrm{n}=9$; IRF4 $4^{--}$plus TNBS and hyper-IL-6, $\mathrm{n}=6$; IRF4 ${ }^{-/}$control, $\mathrm{n}=4$ ). $(\mathbf{C}) \mathrm{TNBS- \text {treated }}$ $\mathrm{IRF}^{-/-}$mice exhibited severe inflammation upon hyper-IL-6 treatment that was indistinguishable from colitis in the wild-type control mice. High-resolution mini-endoscopy consistently demonstrated severe mucosal inflammation upon hyper-IL-6 treatment in the IRF4 knockout mice that was comparable to the colitis activity in the wild-type mice. Representative histological and endoscopic images from the different groups of mice in TNBS-induced colitis are shown. (D) Statistical evaluation of histological and endoscopic results corresponding to the pictures in Fig. 1C.

an endoscopic analysis of colitis severity (Fig. 1C). Whereas the colonic mucosa of the IRF4 $4^{-/-}$mice was unaffected, the wild-type mice showed severe inflammation of the mucosa upon TNBS application. The histological assessment of colitis severity consistently showed significantly higher inflammation scores in the wild-type mice than in the IRF4-deficient mice and the mean score in the latter was similar to that of the healthy untreated control mice. In contrast, the administration of hyper-IL-6 led to severe mucosal lesions, including large ulcers in the TNBS-treated IRF4 ${ }^{-/}$mice (Fig. 1D).

\section{Decreased production of IL-6 and IL-17 in TNBS-treated IRF4-deficient mice}

To clarify the proinflammatory mechanisms inducing colitis in the protected IRF4 phenotype, we focused on several proinflammatory factors. On the one hand we evaluated TNBS-treated IRF4 ${ }^{-/-}$and wild-type mice and on the other the effect of hyper-IL-6 application in the otherwise protected IRF4-deficient mice on the expressions of proinflammatory factors in the long-term course.

Interestingly, there was no difference in mRNA expression of the Th1 and Th2 master regulator transcription factors T-bet and GATA-3 between the IRF4 ${ }^{-/}$and wild-type mice upon TNBS administration in the long term course (Fig. 2A and B). The application of hyper-IL-6 had no influence on T-bet and GATA-3 expression. Importantly, hyper-IL-6 itself induced IL-6 production in the TNBS-treated IRF4-deficient mice (Fig. 2C). Although IL-6 mRNA production was lower than that in the TNBS-treated wild-type mice, it was significantly induced compared with the TNBS-treated IRF-deficient mice without hyper-IL-6 administration (Fig. 2C). The analysis of IL-17 expression revealed a significant difference between TNBS-treated IRF4-deficient mice and wild-type mice (Fig. 2D). In the 


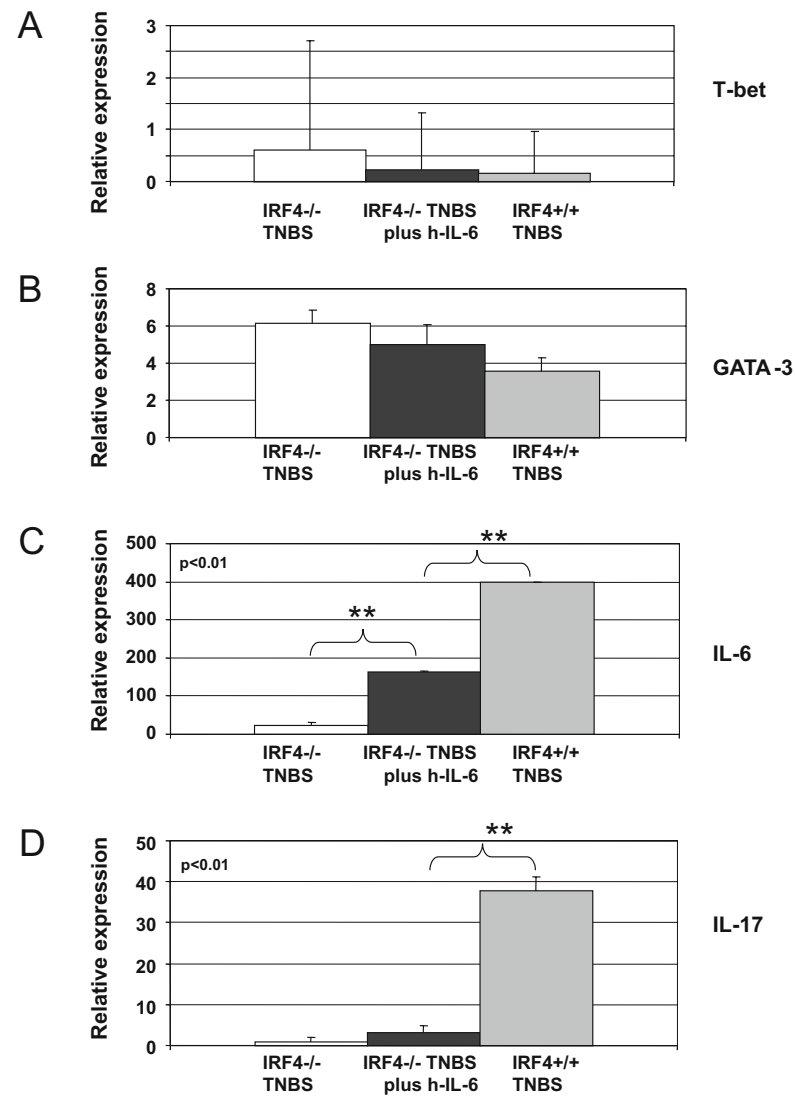

Fig. 2. Measurement of characteristic T-cell cytokines/transcription factors in TNBS-treated wild-type and $\mathrm{IRF}^{-/-}$mice. The mRNA expression of the master regulator of Th1 cells T-bet as well as the mRNA expression of GATA-3 in the colons of the TNBS-treated IRF4-deficient and the wild-type mice were analyzed by quantitative PCR ( $n=6$ per group). We found no significant differences in the expression levels of T-bet and GATA-3 between the groups (A, B). Upon administration of hyper-IL-6, neither T-bet nor GATA-3 mRNA was induced (A, B, gray bar). IL-6 mRNA was strongly reduced in the IRF4 ${ }^{-/}$mice compared with the wild-type mice (C). The same effect was observed analyzing IL-17 mRNA expression. The application of hyper-IL-6 induced IL-6 expression, but not IL-17 (D, gray bar).

** Significance between the groups $(\mathrm{p}<0.01)$.

IRF4 $4^{-/}$mice, IL-17 expression was strikingly reduced compared with that in the wild-type mice. Furthermore, we found that IL-17 was not induced upon hyper-IL-6 administration, suggesting that the underlying mechanism is an intrinsic defect in IL-17 production.

IRF4 ${ }^{-/-}$reconstituted immunodeficient $R A G 2^{-/-}$mice are protected from colitis, but exhibit "minimal" disease in the long-term course

To study the cytokine pattern in a second $\mathrm{T}$ cell-dependent experimental colitis model in the long-term course, we determined the effects of IRF4 in a transfer colitis model in which $\mathrm{CD} 4{ }^{+} \mathrm{CD} 45 \mathrm{RB}^{\text {high }} \mathrm{T}$ cells are adoptively transferred into immunocompromised hosts. Splenic CD45RB ${ }^{\text {high }} \mathrm{CD}^{+}$cells were isolated either from wild-type or $\mathrm{IRF}^{-/-}$mice. The cells were then intraperitoneally injected into immunodeficient $\mathrm{RAG}^{-/-}$mice. Within 20 days the RAG mice reconstituted with $\mathrm{CD}_{45 \mathrm{RB}^{\text {high }}} \mathrm{CD}^{+}{ }^{+} \mathrm{T}$ cells from wild-type mice showed diarrhea and lost body weight (Fig. 3A). In contrast, $\mathrm{RAG}^{-/}$mice reconstituted with $\mathrm{CD} 45 \mathrm{RB}^{\text {high }}$ $\mathrm{CD} 4^{+} \mathrm{T}$ cells from IRF4 $4^{-/-}$mice gained weight (Fig. 3A). Interestingly, $\mathrm{IRF}^{-/-}$reconstituted $\mathrm{RAG} 2^{-/}$mice and $\mathrm{RAG2}^{-/-}$control mice exhibited no differences in body weight until day 100 . At this time the RAG2 $2^{--}$control mice continued to gain weight, whereas the IRF4-/reconstituted mice stopped gaining weight. However, the difference in body weight was significant between the wild-type reconstituted mice and the IRF4 $4^{-/-}$reconstituted mice. Colonoscopy of the mice in the latter group consistently revealed no signs of mucosal inflammation, whereas the RAG2 ${ }^{-/}$deficient mice reconstituted with wild-type $C D 45 R^{\text {high }} \mathrm{CD} 4^{+} \mathrm{T}$ cells exhibited severe mucosal inflammation (Fig. 3B, C). Moreover, the latter mice showed a significantly higher histological score of colitis activity than the former (Fig. 3B).

\section{Reduced IL-6 and IL-17 production in RAG knockout mice reconstituted with IRF4-/- $T$ cells in the long-term course}

We next analyzed cytokine expression in the RAG2-/recipient mice reconstituted with $\mathrm{CD} 45 \mathrm{RB}^{\text {high }} \mathrm{CD}^{+}$ cells from either wild-type or IRF4 $4^{-/-}$mice. Whereas the expressions of various proinflammatory cytokines, such as IL-4, IFN- $\gamma$, IL- $1 \alpha$, and IL- $1 \beta$, were reduced in the $\mathrm{IRF}^{-/-}$reconstituted $\mathrm{RAG}^{-/-}$mice compared with the wild-type reconstituted $\mathrm{RAG}^{-/-}$mice, little or no changes in the expressions of IL- 10 and TNF- $\alpha$ were noted (Fig. 4A). However, further analysis of cytokine expression in the gut revealed a marked reduction in mucosal IL-6 expression (Fig. 4A) in the RAG knockout mice reconstituted with IRF4-deficient cells compared with the mice given wild-type T cells. Furthermore, isolated spleen cells derived from the IRF4 ${ }^{-/-}$reconstituted $\mathrm{RAG}^{-/-}$mice showed significantly lower levels of IL-6 and IL-17 production than cells from the wild-type reconstituted $\mathrm{RAG}^{-/}$mice (Fig. 4B).

\section{DISCUSSION}

In this study we analyzed two experimental models of colitis in its long-term course. TNBS-treated mice were evaluated on day 5 and $C D 45 \mathrm{RB}^{\text {high }}$ reconstituted mice were evaluated on day 119. In the latter case the time span was increased fivefold compared with the evaluation procedure that we usually use in transfer colitis (15 to 20 days). In prior studies, TNBS colitis was evaluated on days 3 or 4 . Compared with these studies (Mudter et al. 2008), TNBS-treated IRF4 $4^{-/}$mice tended to recover from inflammation on days 4 and 5 in our current experiments. Since we know that IL-6 might play a role in acute and chronic inflammation, we aimed to analyze cytokines and proinflammatory factors in the 

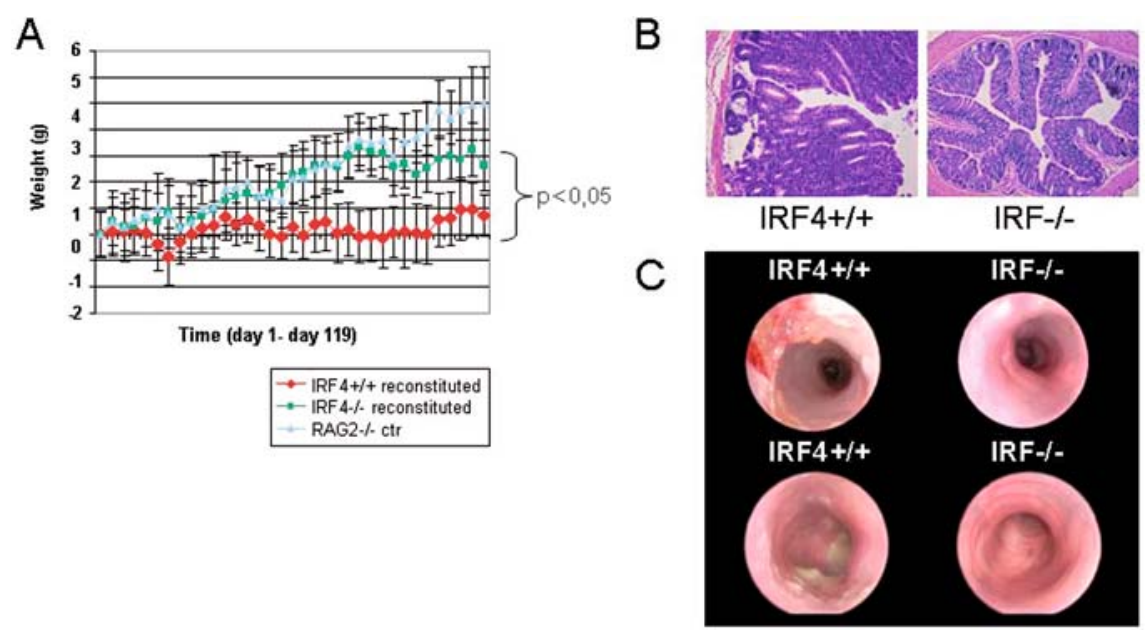

Fig. 3. Protective effect of IRF4 $4^{-/-}$in long-term transfer colitis. (A) CD45RB high $\mathrm{CD} 4^{+} \mathrm{T}$ cells from wild-type or IRF4 ${ }^{-/-}$mice were adoptively transferred into immunodeficient $\mathrm{RAG}^{-/-}$mice. $\mathrm{RAG}^{-/-}$mice reconstituted with wild-type $\mathrm{T}$ cells developed severe colitis accompanied by weight loss (red curve). After initial weight loss from day 13 to day 17, body weight remained static in the state of chronic inflammation. In contrast, mice given IRF4 $4^{--} \mathrm{T}$ cells were completely protected and even gained body weight (green curve) similar to RAG2 $2^{--}$control mice (blue curve). The differences between the two reconstituted groups were significant at days 13 to $17(\mathrm{p}<0.01)$ and from day 25 until evaluation of the mice at day 119. ( $\mathrm{n}=9$ mice per group, three independent experiments). (B) Histological scoring revealed significantly reduced colitis activity in $\mathrm{IRF}^{-/-}$reconstituted mice compared to IRF4 ${ }^{+/+}$reconstituted mice $(\mathrm{p}<0.01)$. (C) Endoscopy consistently showed an inflamed mucosa in the RAG2 knockout mice given wild-type T cells, whereas little or no inflammation was noted in the RAG2 $2^{-/-}$mice reconstituted with IRF4-deficient $\mathrm{T}$ cells.

late phase of colitis (Atreya et al. 2000). Indeed, we found that IL-6 was significantly increased in TNBS-treated wild-type mice compared with IRF4-deficient mice on day 5 . To focus on Th1 regulatory and counter-regulatory mechanisms, we analyzed GATA-3 and T-bet expression. The transcription of the GATA-3 gene may be induced by IRF4 (Lohoff and Mak 2005) and strongly contributes to Th2 differentiation. Thus we measured GATA-3 expression and could show that in Th1 colitis, independent of IRF4 deficiency, GATA-3 expression is not differentially regulated. The transcription factor T-bet is known to regulate early Th1 responses in experimental colitis (Neurath et al. 2002). It was shown that T-bet is overexpressed before colitis symptoms occur and that overexpression of T-bet may accelerate experimental colitis. In this study it was found that retroviral transduction of $\mathrm{T}$-bet in $\mathrm{CD}_{2} \mathrm{~L}^{+} \mathrm{CD} 4^{+}$ $\mathrm{T}$ cells exacerbated colitis in reconstituted SCID mice. In contrast, $\mathrm{T}$-bet-deficient $\mathrm{T}$ cells failed to induce colitis in adoptive transfer experiments, suggesting that overexpression of T-bet is essential and sufficient to promote early Th1-mediated colitis in vivo. In our current studies we found that neither T-bet nor GATA-3, the master Th2 transcription factor, were differentially expressed in IRF4-deficient and wild-type mice in longterm colitis, suggesting that IL-6 might dominate chronic inflammation (Neurath et al. 2002).

The lack of IL-6 production and the consecutive protective phenotype of IRF4 $4^{-/}$mice could be reconstituted by administration of hyper-IL-6. Interestingly, this effect persisted after one single hyper-IL-6 application. This time span is longer than the half-life of this protein. This long-lasting effect demonstrates that IL-6 and its soluble receptor are effective fomenters of inflammation. Focusing on the underlying mechanism, there is great evidence that IL-6 and its soluble receptor itself induce IL-6 production, thus promoting inflammation at least to a certain extent. Since it is known that IL-6 contributes to the differentiation of Th17 cells, which in turn may activate IL-6 signaling (Ivanov et al. 2006; Mudter and Neurath 2007; Ogura et al. 2008), we analyzed the effect of hyper-IL-6 application on IL-17 induction. Surprisingly, IL-17 was not induced in IRF4 ${ }^{-/-}$ mice upon hyper-IL-6 treatment, suggesting that there is an intrinsic defect in IL-17 production in IRF4-deficient mice. The lack of IL-17 in IRF4-deficient mice was previously described (Brustle et al. 2007). However, the molecular mechanism is still unclear, although there is strong evidence that IRF4 directly activates IL-17 production on the transcriptional level (Mudter et al., unpublished data). Previous studies by other groups strongly suggest that IL-17 is an important mediator of inflammation (Brustle et al. 2007; Ivanov et al. 2006; Yen et al. 2006). It was also postulated that IL-17a triggers a positive-feedback loop of IL-6 signaling (Ogura et al. 2008). These data highlight the important role of IL-6 as both an inducer and an effector cytokine of Th17 cells. We have shown that the lack of IL-17 is not a "conditio sine qua non" in chronic long-term colitis because it is sufficient to reconstitute the lack of IL-6 to induce colitis in otherwise protected IRF4-deficient mice. Importantly, it would be necessary to reconstitute IL-17 production via application of IL-17 into IRF4 $4^{-/}$mice to observe the proinflammatory effect of IL-17 (manuscript in preparation). With regard to therapeutic options it might be helpful to attribute the duration of 
A

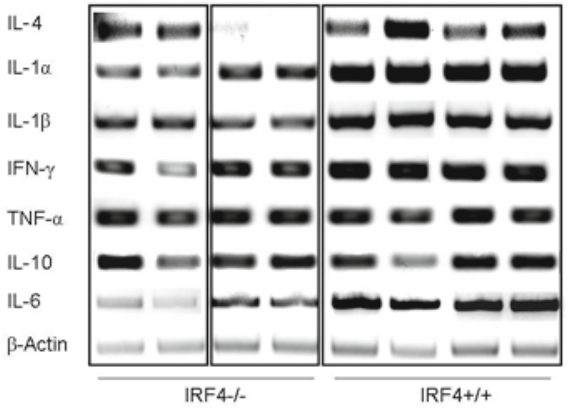

B

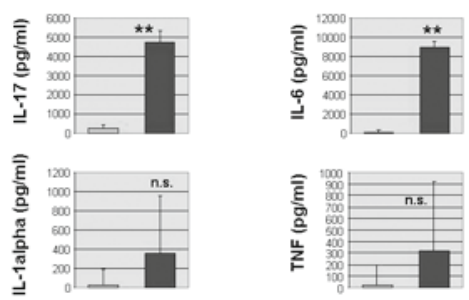

Fig. 4. Reduced IL-6 and IL-17 production in RAG knockout mice reconstituted with $\mathrm{IRF}^{-/-} \mathrm{T}$ cells. (A) RAG recipient mice either reconstituted with $\mathrm{CD}_{45} \mathrm{RB}^{\text {high }} \mathrm{CD}^{+}$cells from wild-type or IRF4 ${ }^{-/}$ice were analyzed by colon specimen and semiquantitative PCR. Whereas the expressions of various proinflammatory cytokines, such as IL- 4 , IFN- $\gamma$, IL- $1 \alpha$, and IL- $1 \beta$, were reduced in the latter compared with the former mice, little or no changes in the expressions of IL-10 and TNF- $\alpha$ were noted. (B) Splenic cells from reconstituted $\mathrm{RAG}^{-/-}$mice $(\mathrm{n}=9)$ were isolated and incubated for $48 \mathrm{~h}$ and the supernatants were measured using ELISA. Analysis of cytokine expression revealed a marked reduction in IL-6 production as well as an absent expression of IL-17 in the RAG2 knockout mice reconstituted with IRF4-deficient cells compared with the mice given wild-type T cells. The differences in IL-1 $\alpha$ and TNF production were not significant. Data are shown as mean values \pm SEM from three independent experiments.

** Significance between the groups $(\mathrm{p}<0.01)$, n.s. - non significant.

disease or current stage of the immune response to different cytokine profiles. Thus a stage-specific antagonistic therapy might be designed.

Acknowledgment: The work of M. F. Neurath and J. Mudter was supported by a grant from the Stiftung Rheinland-Pfalz für Innovation.

\section{REFERENCES}

Atreya R, Mudter J, Finotto S et al (2000) Blockade of interleukin 6 trans signaling suppresses $\mathrm{T}$-cell resistance against apoptosis in chronic intestinal inflammation: evidence in crohn disease and experimental colitis in vivo. Nat Med 6:583-588

Autenrieth IB, Bucheler N, Bohn E et al (1997) Cytokine mRNA expression in intestinal tissue of interleukin-2 deficient mice with bowel inflammation. Gut 41:793-800

Blumberg RS, Saubermann LJ, Strober W (1999) Animal models of mucosal inflammation and their relation to human inflammatory bowel disease. Curr Opin Immunol 11:648-656
Boirivant M, Fuss IJ, Chu A et al (1998) Oxazolone colitis: A murine model of $\mathrm{T}$ helper cell type 2 colitis treatable with antibodies to interleukin 4. J Exp Med 188:1929-1939

Brustle A, Heink S, Huber M et al (2007) The development of inflammatory $\mathrm{T}(\mathrm{H})-17$ cells requires interferon-regulatory factor 4. Nat Immunol 8:958-966

Ivanov II, McKenzie BS, Zhou L et al (2006) The orphan nuclear receptor RORgammat directs the differentiation program of proinflammatory IL-17+ $\mathrm{T}$ helper cells. Cell 126:1121-1133

Lohoff M, Mak TW (2005) Roles of interferon-regulatory factors in T-helper-cell differentiation. Nat Rev Immunol 5:125-135

Lohoff M, Mittrucker HW, Prechtl S et al (2002) Dysregulated $\mathrm{T}$ helper cell differentiation in the absence of interferon regulatory factor 4. Proc Natl Acad Sci USA 99:11808-11812

Macdonald TT, Monteleone G (2005) Immunity, inflammation, and allergy in the gut. Science 307:1920-1925

Morrissey PJ, Charrier K, Braddy S et al (1993) CD4+ T cells that express high levels of $\mathrm{CD} 45 \mathrm{RB}$ induce wasting disease when transferred into congenic severe combined immunodeficient mice. Disease development is prevented by cotransfer of purified CD4+ T cells. J Exp Med 178:237-244

Mudter J, Amoussina L, Schenk M et al (2008) The transcription factor IFN regulatory factor- 4 controls experimental colitis in mice via T cell-derived IL-6. J Clin Invest 118:2415-2426

Mudter J, Neurath MF (2003) Mucosal T-cells: mediators or guardians of inflammatory bowel disease? Curr Opin Gastroenterol 19:343-349

Mudter J, Neurath MF (2007) IL-6 signaling in inflammatory bowel disease: pathophysiological role and clinical relevance. Inflamm Bowel Dis 13:1016-1023

Mudter J, Wirtz S, Galle PR et al (2002) A new model of chronic colitis in SCID mice induced by adoptive transfer of CD62L + CD4 + T cells: Insights into the regulatory role of interleukin-6 on apoptosis. Pathobiology 70:170-176

Neurath MF, Fuss I, Kelsall BL et al (1995) Antibodies to interleukin 12 abrogate established experimental colitis in mice. J Exp Med 182:1281-1290

Neurath MF, Weigmann B, Finotto S et al (2002) The transcription factor $\mathrm{T}$-bet regulates mucosal $\mathrm{T}$ cell activation in experimental colitis and Crohn's disease. J Exp Med 195:1129-1143

Ogura H, Murakami M, Okuyama Y et al (2008) Interleukin-17 promotes autoimmunity by triggering a positive-feedback loop via interleukin-6 induction. Immunity 29:628-636

Podolsky DK (2002) Inflammatory bowel disease. N Engl J Med 347:417-429

Powrie F, Leach MW, Mauze S et al (1993) Phenotypically distinct subsets of CD4+ T cells induce or protect from chronic intestinal inflammation in C. B-17 scid mice. Int Immunol 5:1461-1471

Rengarajan J, Mowen KA, McBride KD et al (2002) Interferon regulatory factor 4 (IRF4) interacts with NFATc2 to modulate interleukin 4 gene expression. J Exp Med 195:1003-1012 
Sartor RB (2004) Therapeutic manipulation of the enteric microflora in inflammatory bowel diseases: antibiotics, probiotics, and prebiotics. Gastroenterology 126:1620-1633

Shanahan F (2002) Crohn's disease. Lancet 359:62-69

Strober W, Fuss IJ, Blumberg RS (2002) The immunology of mucosal models of inflammation. Annu Rev Immunol 20:495-549

Wehkamp J, Schmid M, Fellermann K et al (2005) Defensin deficiency, intestinal microbes, and the clinical phenotypes of Crohn's disease. J Leukoc Biol 77:460-465
Wirtz S, Becker C, Blumberg R et al (2002) Treatment of $\mathrm{T}$ cell-dependent experimental colitis in SCID mice by local administration of an adenovirus expressing IL-18 antisense mRNA. J Immunol 168:411-420

Yen D, Cheung J, Scheerens H et al (2006) IL-23 is essential for $\mathrm{T}$ cell-mediated colitis and promotes inflammation via IL-17 and IL-6. J Clin Invest 116:1310-1316 